\title{
The presence of lepidic and micropapillary/solid pathological patterns as minor components has prognostic value in patients with intermediate-grade invasive lung adenocarcinoma
}

\author{
Yucheng Hou ${ }^{1 \#}$, Weijian Song ${ }^{1 \#}$, Mingzhi Chen ${ }^{2}$, Jianfeng Zhang ${ }^{1}$, Qingquan Luo ${ }^{1}$, Sang-Won Um ${ }^{3}$, \\ Francesco Facchinetti ${ }^{4}$, Stefano Bongiolatti ${ }^{5}$, Qianjun Zhou ${ }^{1}$
}

${ }^{1}$ Department of Thoracic Surgery, Shanghai Lung Cancer Center, Shanghai Chest Hospital, School of Medicine, Shanghai Jiao Tong University, Shanghai, China; ${ }^{2}$ Department of Cardiothoracic Surgery, The 72th Group Army Hospital of People's Liberation Army, Huzhou, China; ${ }^{3}$ Division of Pulmonary and Critical Care Medicine, Department of Medicine, Samsung Medical Center, Sungkyunkwan University School of Medicine, Seoul, Korea; ${ }^{4}$ Université Paris-Saclay, Institut Gustave Roussy, Inserm, Biomarqueurs Prédictifs et Nouvelles, Stratégies Thérapeutiques en Oncologie, Villejuif, France; ${ }^{5}$ Thoracic Surgery Unit, Careggi University Hospital, Florence, Italy

Contributions: (I) Conception and design: Y Hou, W Song, Q Zhou; (II) Administrative support: Q Luo, Q Zhou; (III) Provision of study materials or patients: Y Hou, W Song, Q Luo, Q Zhou; (IV) Collection and assembly of data: Y Hou, W Song, M Chen, J Zhang; (V) Data analysis and interpretation: All authors; (VI) Manuscript writing: All authors; (VII) Final approval of manuscript: All authors.

\#These authors contributed equally to this work.

Correspondence to: Qianjun Zhou. Department of Thoracic Surgery, Shanghai Lung Cancer Center, Shanghai Chest Hospital, Shanghai Jiao Tong University, Shanghai, China. Email: zhouqj@sjtu.edu.cn.

Background: The acinar- and papillary-predominant histological subtypes are the most common types of invasive lung adenocarcinoma and are considered "intermediate-grade" carcinomas with heterogeneous prognosis. This study investigated the prognostic significance of the lepidic and micropapillary/solid pathological patterns as minor components in patients with intermediate-grade lung adenocarcinomas.

Methods: A total of 697 patients with pathological N0M0 acinar/papillary-predominant lung adenocarcinomas $\leq 3 \mathrm{~cm}$ in diameter, who underwent curative resection in our institution between June 1, 2014 and August 31, 2016, were retrospectively enrolled in this study. Acinar/papillary-predominant lung adenocarcinomas were classified into four subtypes according to the presence of the minor pathological components lepidic (Lep), micropapillary (MP), and solid (S). The subtypes were MP/S'Lep ${ }^{+}, \mathrm{MP}^{-} \mathrm{S}^{-} \mathrm{Lp}^{-}$, $\mathrm{MP} / \mathrm{S}^{+} \mathrm{Lep}^{+}$, and MP/S Lep ${ }^{-}$. The 5 -year recurrence-free survival (RFS) and overall survival (OS) were recorded. Factors affecting survival were analyzed by Cox regression method.

Results: Among 697 intermediate-grade lung adenocarcinomas, the distribution of patients was as follows: MP/S ${ }^{-}$ep $^{+}$type $(\mathrm{n}=314 ; 45.0 \%), \mathrm{MP}^{-} \mathrm{S}^{-} \mathrm{Lep}^{-}$type $(\mathrm{n}=144 ; 20.7 \%), \mathrm{MP} / \mathrm{S}^{+} \mathrm{Lep}^{+}$type $(\mathrm{n}=133 ; 19.1 \%)$, and MP/S Lep ${ }^{-}$type (n=106; 15.2\%). The 5-year RFS rates were 98.7\%, 94.4\%, 94.0\%, and 81.9\%, respectively $(\mathrm{P}<0.001)$. The 5-year OS rates were 98.4\%, 94.4\%, 96.6\%, and 87.7\%, respectively $(\mathrm{P}<0.001)$. Multivariate analysis revealed that the MP/S ${ }^{+} \mathrm{Lep}^{-}$subtype was an independent poor prognostic factor of both RFS and OS.

Conclusions: Acinar/papillary-predominant adenocarcinoma is an "intermediate-grade" carcinoma that can be further classified into subtypes according to the presence of lepidic and micropapillary/solid pathological patterns with significantly different prognosis. This classification may be useful in evaluating the recurrence risk and guiding adjuvant therapies in patients with acinar/papillary-predominant stage I lung adenocarcinoma.

Keywords: Adenocarcinoma; acinar; papillary; prognosis; minor pathological component patterns

^ ORCID: 0000-0002-8326-2043. 
Submitted Oct 15, 2021. Accepted for publication Dec 24, 2021.

doi: $10.21037 /$ tlcr-21-934

View this article at: https://dx.doi.org/10.21037/tlcr-21-934

\section{Introduction}

In 2015, the World Health Organization classified invasive lung adenocarcinomas into three groups according to peculiar pathological and clinical features. The "lowgrade" group is represented by the lepidic pattern, the "intermediate-grade" group is represented by the acinar and papillary patterns, and the "high-grade" group is characterized by solid and micropapillary patterns $(1,2)$. Most adenocarcinomas are composed of more than one pathological component, while pure adenocarcinomas with a single pathological pattern are rare (3).

It is well known that lepidic-predominant lung adenocarcinomas are associated with a good prognosis $(4,5)$. Conversely, micropapillary- or solid-predominant subtypes are associated with poorer survival (6-9). Acinarand papillary-predominant adenocarcinoma is the most common subtype (50-70\%) of invasive adenocarcinomas, with a heterogeneous prognosis (10-12). Due to the high heterogeneity of the "intermediate-grade" group, its prognostic factors have not been well-studied.

Based on semiquantitative analysis of histology, previous investigations have focused on the impact of the second predominant pattern (SPP) following the predominant pattern on the survival and recurrence in patients with "intermediate-grade" adenocarcinomas. Yanagawa reported that patients with a solid or micropapillary pattern as a minor component had poor prognosis (13). However, recent findings from a large European database study concluded that there was no difference in recurrence-free survival (RFS) according to the SPP $(\mathrm{P}=0.522)(14)$. We hypothesize that every single pathological component of the cancer that is present is one of the important reasons for the heterogeneity of "intermediate-grade" adenocarcinomas and lead to different prognosis, rather than just one or two major components.

This study investigated the prognostic factors of acinar/ papillary-predominant lung adenocarcinomas and stratified this "intermediate-grade" group of lung adenocarcinomas according to the presence of the lepidic and micropapillary/ solid pathological patterns. We present the following article in accordance with the STROBE reporting checklist (available at https://tlcr.amegroups.com/article/ view/10.21037/tlcr-21-934/rc).

\section{Methods}

\section{Patients}

All procedures performed in this study involving human participants were in accordance with the Declaration of Helsinki (as revised in 2013). This retrospective study was approved by the Institutional Review Board of the Shanghai Chest Hospital (No. KS2011). Individual consent for this retrospective analysis was waived. Patients with pathologically confirmed acinar/papillarypredominant adenocarcinomas $\leq 3 \mathrm{~cm}$ in diameter who underwent curative resection between June 1, 2014 and August 31, 2016 were retrospectively reviewed. Clinical and pathological characteristics were collected, including age, gender, smoking history, body mass index (BMI), American Society of Anesthesiologists (ASA) index, cancer history (except lung cancer), tumor size, pathological $\mathrm{T}(\mathrm{pT})$ stage, tumor location, predominant histological subtype, surgical technique, resection type, epidermal growth factor receptor $(E G F R)$ gene status, and the following administration of adjuvant chemotherapy. The smokers were grouped into two groups: mild ( $<20$ packyears) and heavy ( $\geq 20$ pack-years). Patients were excluded if they underwent neoadjuvant therapy, had multiple lung lesions, or received palliative/diagnostic excision surgery. To avoid the influence of lymph node metastasis, only pN0 patients were selected for subsequent analysis.

Surgery was conducted either by minimally invasive surgery (MIS), including video-assisted thoracic surgery (VATS) and robotic-assisted thoracic surgery (RATS), or thoracotomy. Lobectomy with systemic lymph node dissection was routinely performed. Sublobar resection, including segmentectomy and wedge resection, was performed for peripheral lesions with ground-glass appearance on computed tomography (CT) scan.

All patients were diagnosed according to the 2011 ATS/ERS/IASLC classification, and each subtype of adenocarcinoma was recorded in $5 \%$ increments by two independent senior pathologists in our institution. Any disagreements between the pathologists were resolved via 
discussion. All tumors were staged according to the $8^{\text {th }}$ edition UICC TNM classification.

\section{Subtyping according to the presence of lepidic and micropapillary/solid pathological patterns}

The acinar/papillary-predominant adenocarcinomas were classified according to the presence of lepidic and micropapillary/solid pathological patterns. If the lepidic (Lep) component is present and the micropapillary (MP)/solid (S) component is absent, the case was classified as the MP/S ${ }^{-} \mathrm{Lep}^{+}$subtype. If none of the MP/S or Lep components are present, the case was classified as the MP/S'Lep ${ }^{-}$subtype. If both the MP/S component and the Lep component are present, the case was classified as the $\mathrm{MP} / \mathrm{S}^{+} \mathrm{Lep}^{+}$subtype. If the MP/S component is present and the Lep component is absent, the case was classified as the MP/S $\mathrm{Sep}^{-}$subtype. The representative pathological pictures were shown in Figure S1.

\section{Follow-up and study endpoints}

The follow-up data were retrieved from the medical records of each patient. All patients were followed up at our hospital or other institution every 3 months for the first 2 years after surgery and every 6 months thereafter for 5 years. A thoracic CT scan and an abdominal CT scan or ultrasonography was routinely performed at each scheduled visit. During follow-up, bone scans and cranial CT/magnetic resonance imaging (MRI) were performed as clinically indicated in patients with bone pain or neurological symptoms, respectively. A positron emission tomography (PET)-CT scan was suggested if the patient had suspected recurrence or metastasis. Patients were also followed up by telephone interview. The cause of death was determined based on medical records or interviews.

The primary endpoint of the study was to evaluate the impact of the presence of the Lep and MP/S pathological patterns on RFS and OS.

\section{Statistical analysis}

Data were presented as number (\%) or mean \pm standard deviation. Analysis of variance (ANOVA) was used to analyze normally distributed continuous variables and the Kruskal-Wallis test was used to analyze continuous variables that did not conform to normal distribution. The $\chi^{2}$ test or Fisher's exact test, as appropriate, was used to assess categorical variables. The Kaplan-Meier method was used to calculate RFS and OS, and the differences between groups were evaluated with Log-rank test. Univariate and multivariable Cox proportional-hazards regression analysis was used to evaluate the prognostic value of various factors. A two-sided $P$ value less than 0.05 was considered statistically significant. All statistical analyses were performed using $\mathrm{R}$ software (version 3.5.2, https://www.r-project.org/).

\section{Results}

\section{Basic characteristics in each patient subtype classified according to the presence of lepidic and micropapillary/ solid pathological patterns}

A total of 697 patients with acinar/papillary-predominant adenocarcinomas were eligible in this study, including 268 $(38.5 \%)$ males and $429(61.5 \%)$ females, with a mean age of 60.8 (Table 1). There were 152 (21.8\%) smokers. In 55 $(7.9 \%)$ cases, patients had a prior history of cancer other than lung cancer, but radical treatment was performed prior to this operation. For surgical techniques, 618 (88.7\%) cases underwent VATS $(\mathrm{n}=580 ; 83.2 \%)$ and RATS $(\mathrm{n}=38$; $5.5 \%)$, while open surgery was performed in $79(11.3 \%)$ patients. Lobectomy was performed in 565 cases $(81.1 \%)$, segmentectomy was performed in $58(8.3 \%)$ cases, and 74 (10.6\%) patients underwent wedge resection.

The patient characteristics in each different micropapillary/solid subtype were summarized in Table 1. Of the 697 cases, 314 (45.0\%), 144 (20.7\%), 133 (19.1\%), and $106(15.2 \%)$ cases were classified as the MP/S ${ }^{-} \mathrm{Lep}^{+}$subtype, the MP/S ${ }^{-} \mathrm{Lep}^{-}$subtype, the MP/S ${ }^{+} \mathrm{Lep}^{+}$subtype, and the $\mathrm{MP} / \mathrm{S}^{+} \mathrm{Lep}^{-}$subtype, respectively. These four subgroups had comparable age, ASA index, smoking history, cancer history, EGFR status, surgical technique, and resection type, but significantly differed in terms of gender $(\mathrm{P}=0.003)$, BMI $(\mathrm{P}=0.043)$, tumor size $(\mathrm{P}<0.001)$, pT stage $(\mathrm{P}<0.001)$, pathological predominant pattern $(\mathrm{P}=0.018)$, and the use of adjuvant chemotherapy $(\mathrm{P}<0.001)$. The MP/S ${ }^{-} \mathrm{Lep}^{+}$ subgroup was associated with the female gender, smaller tumors, and lower pT stage. Patients presenting with MP/S as the minor pattern were more frequently male, had larger tumors, and higher $\mathrm{pT}$ stage.

\section{Survival analysis}

The median follow-up time was 65 months (range, 52-78 months). In the whole study cohort, the 
Table 1 Clinicopathological characteristics of the different subtypes classified according to the presence of lepidic and micropapillary/solid pathological patterns

\begin{tabular}{|c|c|c|c|c|c|}
\hline Characteristics & $\begin{array}{c}\text { MP/S }{ }^{-} \text {Lep }^{+}, n=314 \\
(45.0 \%)\end{array}$ & $\begin{array}{c}\text { MP/S } \text { Lep }^{-}, \mathrm{n}=144 \\
(20.7 \%)\end{array}$ & 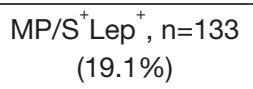 & $\begin{array}{c}\mathrm{MP} / \mathrm{S}^{+} \text {Lep }^{-}, \mathrm{n}=106 \\
(15.2 \%)\end{array}$ & $P$ value \\
\hline Age (mean $\pm S D)$ & $60.4 \pm 9.1$ & $60.7 \pm 8.6$ & $61.1 \pm 10.0$ & $62.0 \pm 8.8$ & 0.457 \\
\hline Male & $103(32.8)$ & $51(35.4)$ & 65 (48.9) & $49(46.2)$ & \\
\hline Female & $211(67.2)$ & $93(64.6)$ & $68(51.1)$ & $57(53.8)$ & \\
\hline $\mathrm{I} / \mathrm{II}$ & $288(91.7)$ & $134(93.1)$ & $125(94.0)$ & $99(93.4)$ & \\
\hline III & $26(8.3)$ & $10(6.9)$ & $8(6.0)$ & $7(6.6)$ & \\
\hline Smoking history, n (\%) & & & & & 0.065 \\
\hline Never & $257(81.9)$ & $116(80.5)$ & $92(69.2)$ & $80(75.5)$ & \\
\hline Yes & $27(8.6)$ & $10(6.9)$ & $9(6.8)$ & $9(8.5)$ & \\
\hline No & $287(91.4)$ & $134(93.1)$ & $124(93.2)$ & $98(92.5)$ & \\
\hline Tumor size, $\mathrm{cm}($ mean $\pm \mathrm{SD})$ & $1.51 \pm 0.56$ & $1.74 \pm 0.58$ & $1.93 \pm 0.57$ & $2.07 \pm 0.52$ & $<0.001^{\star}$ \\
\hline pT stage, n (\%) & & & & & $<0.001^{\star}$ \\
\hline T1a & $79(25.2)$ & $19(13.2)$ & $16(12.0)$ & $3(2.8)$ & \\
\hline $\mathrm{T} 1 \mathrm{~b}$ & $183(58.3)$ & $73(50.7)$ & $55(41.4)$ & $35(33.0)$ & \\
\hline $\mathrm{T} 1 \mathrm{c}$ & $46(14.6)$ & $33(22.9)$ & $39(29.3)$ & $32(30.2)$ & \\
\hline $\mathrm{T} 2 \mathrm{a}^{\#}$ & $6(1.9)$ & 19 (13.2) & $23(17.3)$ & $36(34.0)$ & \\
\hline Open & $33(10.5)$ & $10(6.9)$ & $22(16.5)$ & $14(13.2)$ & \\
\hline Resection type, n (\%) & & & & & 0.072 \\
\hline Lobectomy & $246(78.3)$ & $113(78.5)$ & $112(84.2)$ & $94(88.7)$ & \\
\hline Sublobar resection & $68(21.7)$ & $31(21.5)$ & $21(15.8)$ & $12(11.3)$ & \\
\hline EGFR status, $n(\%)(N=179)$ & & & & & 0.826 \\
\hline Wild & $13(25.5)$ & $6(25.0)$ & $18(31.0)$ & $15(32.6)$ & \\
\hline Mutation & $38(74.5)$ & $18(75.0)$ & $40(69.0)$ & $31(67.4)$ & \\
\hline The use of adjuvant chemotherapy, $n$ (\%) & & & & & $<0.001^{\star}$ \\
\hline Yes & $10(3.2)$ & $13(9.0)$ & $14(10.5)$ & $27(25.5)$ & \\
\hline No & $304(96.8)$ & $131(91.0)$ & $119(89.5)$ & $79(74.5)$ & \\
\hline
\end{tabular}

\#, although only tumors less than $3 \mathrm{~cm}$ were included in this analysis, some of the patients were staged as T2a due to pleural invasion. Significant $\mathrm{P}$ values are annotated with asterisk $\left({ }^{*}\right)$. Lep, lepidic; MP/S, micropapillary/solid; SD, standard deviation; BMI, body mass index; ASA index, American Society of Anesthesiologists index; pT stage, pathological T stage; MIS, minimally invasive surgery; EGFR, epidermal growth factor receptor. 

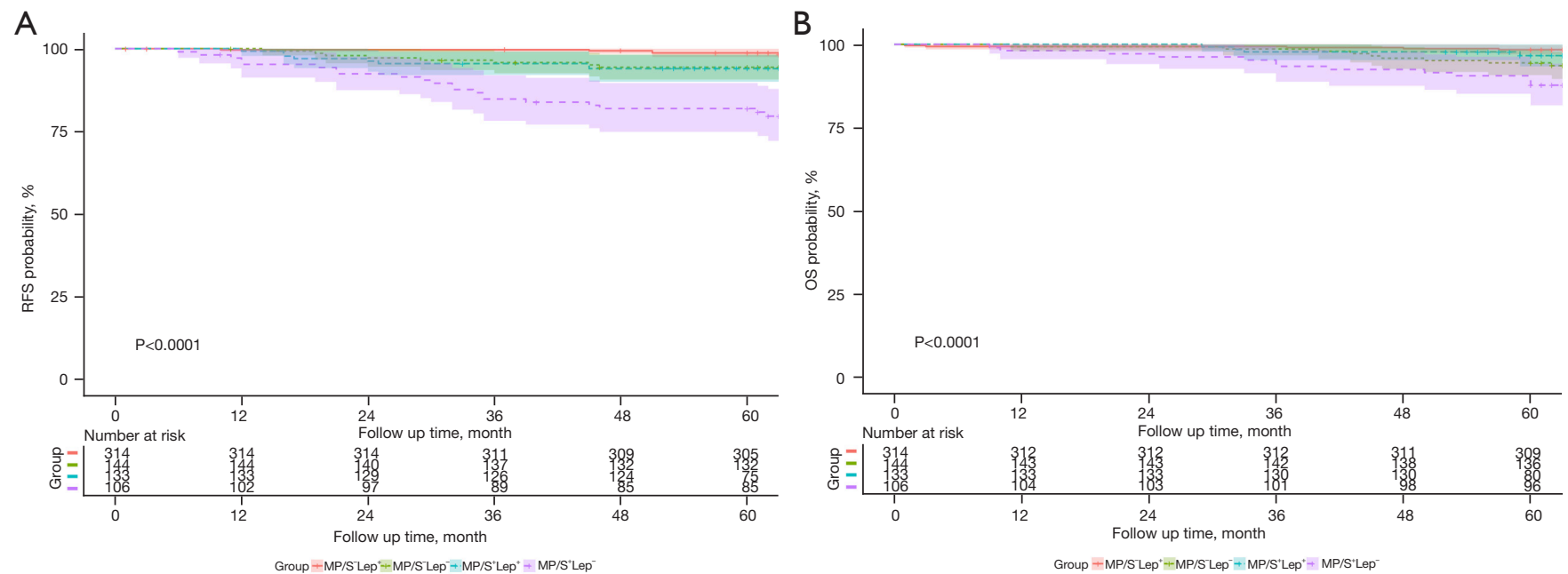

Figure 1 The recurrence-free survival (RFS) curve and the overall survival (OS) curve among the four subgroups (A,B).

Table 2 Pairwise comparisons of the recurrence-free survival among the four subtypes

\begin{tabular}{|c|c|c|c|c|c|c|c|c|c|c|c|c|}
\hline \multirow{2}{*}{ Subgroup } & \multicolumn{12}{|c|}{ Pairwise comparisons using Log-Rank test } \\
\hline & HR & $95 \% \mathrm{Cl}$ & $P$ value & $\mathrm{HR}$ & $95 \% \mathrm{Cl}$ & $P$ value & $\mathrm{HR}$ & $95 \% \mathrm{Cl}$ & $P$ value & $\mathrm{HR}$ & $95 \% \mathrm{Cl}$ & $P$ value \\
\hline MP/S Lep ${ }^{+}$ & - & - & - & 0.28 & $0.09-0.86$ & $0.026^{\star}$ & 0.22 & $0.08-0.67$ & $0.007^{\star}$ & 0.07 & $0.03-0.19$ & $<0.001^{*}$ \\
\hline MP/S Lep ${ }^{-}$ & 3.57 & $1.17-10.91$ & $0.026^{\star}$ & - & - & - & 0.80 & $0.31-2.08$ & 0.650 & 0.26 & $0.11-0.58$ & $0.001^{*}$ \\
\hline MP/S ${ }^{+}$Lep $^{-}$ & 13.96 & $5.26-37.02$ & $<0.001^{*}$ & 3.91 & $1.73-8.83$ & $0.001^{*}$ & 3.14 & $1.44-6.86$ & $0.004^{*}$ & - & - & - \\
\hline
\end{tabular}

Significant $\mathrm{P}$ values are annotated with asterisk $\left({ }^{\star}\right)$. Lep, lepidic; MP/S, micropapillary/solid; HR, hazard ratio; $\mathrm{Cl}$, confidence interval.

5 -year RFS rate and the 5-year OS rate were $94.4 \%$ and $95.6 \%$, respectively. The 5 -year recurrence rate was $5.6 \%$ (39 cases).

There were significant differences in RFS $(\mathrm{P}<0.001)$ and $\mathrm{OS}(\mathrm{P}<0.001)$ among the 4 subgroups. The 5 -year RFS rates of the MP/S ${ }^{-} \mathrm{Lep}^{+}, \mathrm{MP} / \mathrm{S}^{-} \mathrm{Lep}^{-}, \mathrm{MP} / \mathrm{S}^{+} \mathrm{Lep}^{+}$, and $\mathrm{MP} / \mathrm{S}^{+} \mathrm{Lep}^{-}$subtypes were $98.7 \%, 94.4 \%, 94.0 \%$, and $81.9 \%$, respectively (Figure $1 A$ ). The 5 -year OS rates of the $M P / \mathrm{S}^{-} \mathrm{Lep}^{+}, \mathrm{MP} / \mathrm{S}^{-} \mathrm{Lep}^{-}, \mathrm{MP} / \mathrm{S}^{+} \mathrm{Lep}^{+}$, and $\mathrm{MP} / \mathrm{S}^{+} \mathrm{Lep}^{-}$subtypes were $98.4 \%, 94.4 \%, 96.6 \%$, and $87.7 \%$, respectively (Figure $1 B$ ).

Pairwise comparison using the Log-rank test (Tables 2,3) revealed that both the RFS and OS differed significantly between the $\mathrm{MP} / \mathrm{S}^{+} \mathrm{Lep}^{-}$and $\mathrm{MP} / \mathrm{S}^{+} \mathrm{Lep}^{+}$subtypes $(\mathrm{P}<0.01$ and $\mathrm{P}<0.05$, respectively). The $\mathrm{OS}$ of the $\mathrm{MP} / \mathrm{S}^{+} \mathrm{Lep}^{+}$ subtype was comparable to the MP/S ${ }^{-} \mathrm{Lep}^{+}$subtype $(\mathrm{P}=0.27)$. The RFS of the MP/S ${ }^{+} \mathrm{Lep}^{+}$subtype was worse compared to the MP/S ${ }^{-} \mathrm{Lep}^{+}$subtype $(\mathrm{P}<0.007)$. The RFS of the $\mathrm{MP} / \mathrm{S}^{+} \mathrm{Lep}^{-}$subtype was also poorer compared to the MP/S ${ }^{-}$Lep $^{-}$subtype $(\mathrm{P}<0.001)$, while the OS of these 2 subtypes was comparable $(\mathrm{P}=0.146)$.

\section{Prognostic factors in acinar/papillary predominant lung adenocarcinomas}

Univariate analysis suggested that the male gender, age $\geq 70$ years, pleural invasion, the use of adjuvant therapy, and the MP/S ${ }^{+} \mathrm{Lep}^{-}$subtype were associated with poorer RFS, while the MP/S ${ }^{-} \mathrm{Lep}^{+}$subtype was associated with superior RFS (Table 4). Multivariate Cox regression analysis showed that the MP/S ${ }^{+} \mathrm{Lep}^{-}$subtype was the only independent predictor of RFS. The multivariable analysis results of each subtype as a reference are listed in Table S1.

Similarly, univariate analysis demonstrated that the male 
Table 3 Pairwise comparisons of the overall survival among the four subtypes

\begin{tabular}{|c|c|c|c|c|c|c|c|c|c|c|c|c|}
\hline \multirow{2}{*}{ Subgroup } & \multicolumn{12}{|c|}{ Pairwise comparisons using Log-Rank test } \\
\hline & $\mathrm{HR}$ & $95 \% \mathrm{Cl}$ & $P$ value & $\mathrm{HR}$ & $95 \% \mathrm{Cl}$ & $P$ value & $\mathrm{HR}$ & $95 \% \mathrm{Cl}$ & $P$ value & $\mathrm{HR}$ & $95 \% \mathrm{Cl}$ & $P$ value \\
\hline MP/S Lep ${ }^{+}$ & - & - & - & 0.23 & $0.08-0.66$ & $0.007^{*}$ & 0.48 & $0.13-1.78$ & 0.271 & 0.12 & $0.04-0.34$ & $<0.001^{*}$ \\
\hline MP/S'Lep ${ }^{-}$ & 4.42 & $1.51-12.92$ & $0.007^{*}$ & - & - & - & 2.11 & $0.66-6.74$ & 0.208 & 0.54 & $0.24-1.24$ & 0.146 \\
\hline MP/S ${ }^{+}$Lep $^{-}$ & 8.13 & $2.90-22.82$ & $<0.001^{\star}$ & 1.84 & $0.81-4.20$ & 0.146 & 3.89 & $1.27-11.93$ & $0.018^{*}$ & - & - & - \\
\hline
\end{tabular}

Significant $\mathrm{P}$ values are annotated with asterisk (*). Lep, lepidic; MP/S, micropapillary/solid; HR, hazard ratio; Cl, confidence interval.

Table 4 Univariate and multivariate analysis for recurrence-free survival

\begin{tabular}{|c|c|c|c|c|c|c|}
\hline \multirow{2}{*}{ Recurrence-free survival } & \multicolumn{3}{|c|}{ Univariable analysis } & \multicolumn{3}{|c|}{ Multivariable analysis } \\
\hline & HR & $95 \% \mathrm{Cl}$ & $\mathrm{P}$ & HR & $95 \% \mathrm{Cl}$ & $\mathrm{P}$ \\
\hline Male (vs. female) & 1.722 & $0.947-3.132$ & 0.075 & 1.244 & $0.673-2.300$ & 0.486 \\
\hline Age $\geq 70$ years & 2.299 & $1.199-4.408$ & $0.012^{*}$ & 1.774 & $0.912-3.453$ & 0.091 \\
\hline BMI & 1.012 & $0.925-1.110$ & 0.799 & & & \\
\hline ASA III (vs. ASA I/II) & 1.316 & $0.470-3.684$ & 0.601 & & & \\
\hline \multicolumn{7}{|l|}{ pT stage } \\
\hline $\mathrm{T} 1 \mathrm{a}$ & Ref & & & Ref & & \\
\hline $\mathrm{T} 1 \mathrm{~b}$ & 1.025 & $0.277-3.785$ & 0.971 & 0.713 & $0.190-2.670$ & 0.615 \\
\hline $\mathrm{T} 1 \mathrm{c}$ & 4.026 & $1.166-13.908$ & $0.028^{*}$ & 2.034 & $0.565-7.327$ & 0.278 \\
\hline \multicolumn{7}{|l|}{ Smoking history } \\
\hline Never & Ref & & & & & \\
\hline Mild & 1.359 & $0.416-4.438$ & 0.611 & & & \\
\hline Heavy & 1.233 & $0.568-2.676$ & 0.597 & & & \\
\hline Open technique (vs. MIS) & 1.849 & $0.858-3.987$ & 0.117 & & & \\
\hline The use of adjuvant chemotherapy & 4.526 & $2.360-8.681$ & $<0.001^{*}$ & 1.870 & $0.854-4.096$ & 0.118 \\
\hline Sublobar resection (vs. lobectomy) & 0.866 & $0.385-1.946$ & 0.727 & & & \\
\hline \multicolumn{7}{|l|}{ Subtype } \\
\hline
\end{tabular}


Table 5 Univariate and multivariate analysis for overall survival

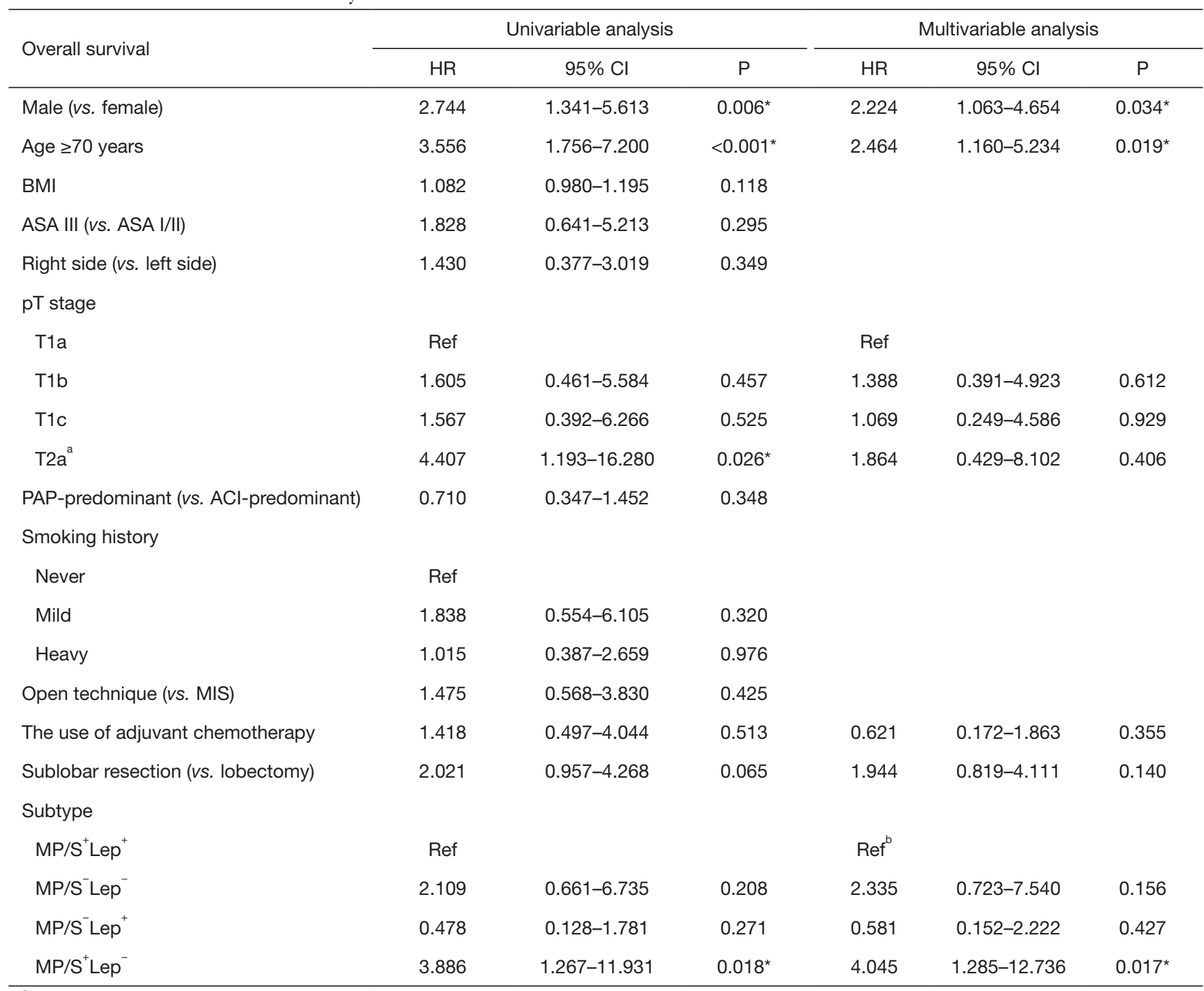

${ }^{\text {a }}$, although only tumors of less than $3 \mathrm{~cm}$ were included in the analysis, some of the patients were staged as T2a because of pleural invasion; ${ }^{b}$, the multivariable analysis results of each subtype as a reference were in Table $S 2$. Significant $P$ values are annotated with asterisk (*). HR, hazard ratio; Cl, confidence; BMI, body mass index; ASA, American Society of Anesthesiologists; pT stage, pathological T stage; PAP-predominant, papillary predominant adenocarcinomas; ACI-predominant, acinar predominant adenocarcinomas; MIS, minimally invasive surgery; Lep, lepidic; MP/S, micropapillary/solid.

gender, age $\geq 70$ years, pleural invasion, and the $\mathrm{MP} / \mathrm{S}^{+} \mathrm{Lep}^{-}$ subtype were associated with poorer OS. Multivariate Cox regression showed that patients aged $\geq 70$ years, the male gender, and the $\mathrm{MP} / \mathrm{S}^{+} \mathrm{Lep}^{-}$subtype were significantly associated with poorer OS (Table 5). The multivariable analysis results of each subtype as a reference are listed in Table S2.

\section{The role of adjuvant chemotherapy in patients with the MP/S Lep subtype}

Since patients with the MP/S ${ }^{+} \mathrm{Lep}^{-}$subtype had relatively poor survival, the role of adjuvant chemotherapy in this subgroup was explored. Adjuvant chemotherapy is more likely to be used in patients with stage IB and thus, the RFS and OS of patients with $\mathrm{MP} / \mathrm{S}^{+} \mathrm{Lep}^{-}$ 

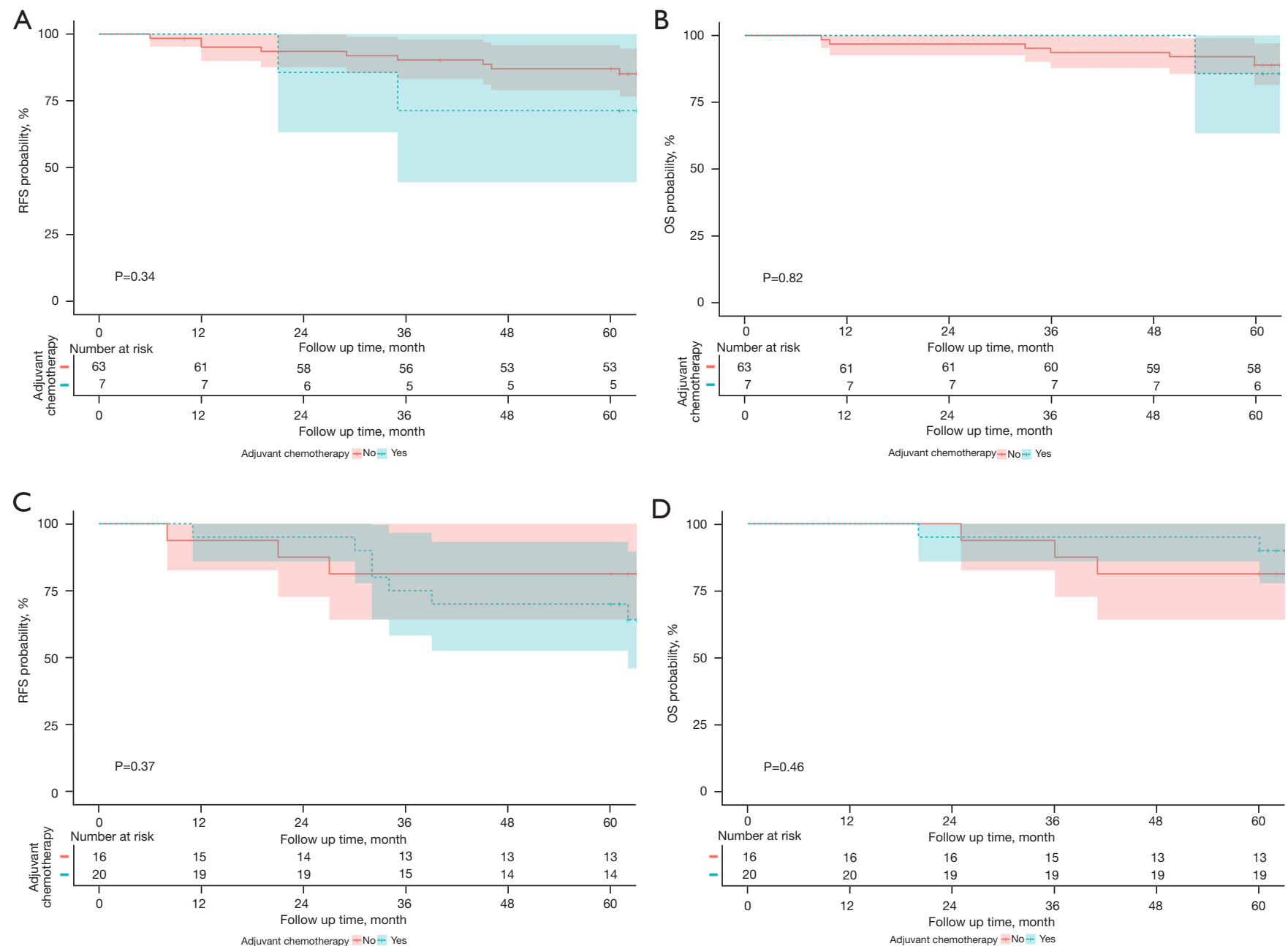

Figure 2 The recurrence-free survival (RFS) and the overall survival (OS) of patients with the MP/S ${ }^{+} \mathrm{Lep}^{-}$subtype receiving adjuvant chemotherapy or not in stage IA (A,B) or stage IB (C,D).

subtype in stage IA (Figure $2 A, 2 B$ ) and IB (Figure 2C,2D), respectively, were analyzed. No significant difference was observed between patients who received adjuvant chemotherapy and patients who did not received adjuvant chemotherapy.

\section{Discussion}

The present study classified lung adenocarcinomas into subgroups according to the presence of lepidic and micropapillary/solid pathological patterns and demonstrated that the presence of minor MP/S and Lep components can be used to stratify the prognosis of patients with acinar/ papillary-predominant adenocarcinoma, which is the most common histological pattern with a heterogeneous prognosis.

Multivariate analysis identified the $\mathrm{MP} / \mathrm{S}^{+} \mathrm{Lep}^{-}$subtype as an independent prognostic factor of both RFS and OS. Although acinar/papillary-predominant adenocarcinomas account for more than $50-70 \%$ of lung adenocarcinomas, there is a paucity of studies investigating its prognostic factors. Since 2004, it has been well accepted that most adenocarcinomas (94\%) present with more than one pathological component (3). According to the WHO classification, the components of up to or more than $5 \%$ of the minor components should be recorded, indicating that each component in the adenocarcinoma might have an effect on the prognosis and not only the predominant component. Previous studies have often focused on the relationship between the SPP and prognosis. However, the 
impact of the SPP remains controversial $(4,11,15)$. A recent European multicenter study found no significant difference in terms of DFS according to SPP (14).

Recently, a new grading system for invasive lung adenocarcinoma based on the predominant and high-grade patterns was proposed. It was noted that tumors with more than $20 \%$ high-grade pattern as the SPP should be classified into a poorly differentiated group as their invasiveness was similar to that of high-grade pattern predominant adenocarcinomas (16). In fact, the SPP might not be a good parameter for certain reasons. In some cases, the SPP cannot be determined when the proportion of secondary components are equal, such as $20 \%$ lepidic pattern and $20 \%$ micropapillary pattern, and this has been excluded in some of previous studies (11). In addition, it can be difficult to accurately quantify each minor pathological component based on semiquantitative analysis, even for the experienced pathologist. During the progression of adenocarcinoma, more aggressive tumors may contain higher grade invasive components such as the MP/S component, accompanied by the disappearance of the Lep component. In this study, the extent of invasiveness in the "intermediate-grade" adenocarcinoma was estimated based on the presence of the lepidic and micropapillary/solid pathological patterns. This does not require the quantification of all the pathological components. To the best of our knowledge, the present study not only examined the largest cohort of patients with "intermediate-grade" lung adenocarcinoma, but is also the first to use the presence of the lepidic and micropapillary/ solid pathological patterns as a prognostic factor to stratify acinar/papillary-predominant stage I lung adenocarcinomas.

The Lep component is a favorable prognostic factor regardless of its percentage $(17,18)$ and the presence of the MP/S component at higher than $5 \%$ indicates a poor prognosis even if it is not the predominant component (19). These data suggested that the presence of Lep and MP/S pathological components, rather than their percentages, is more important for the prognosis of "intermediate-grade" adenocarcinoma. Therefore, in this study, the combination of the Lep and MP/S minor components was used to stratify "intermediate-grade" lung adenocarcinoma. Since "intermediate-grade" adenocarcinomas are all acinar/ papillary-predominant, the acinar/papillary component was not included as a parameter to avoid redundancy.

In the entire study cohort, the 5-year RFS and OS were $94.4 \%$ and $95.7 \%$, respectively, after a median followup period of 65 months. The 5 -year recurrence rate was $5.6 \%$ (39 cases). Age, gender, and tumor size have been recognized as prognostic factors for both RFS and OS in previous studies involving early-stage lung adenocarcinoma $(3,12,14,19,20)$. In this study, the MP/S ${ }^{+} \mathrm{Lep}^{-}$subtype was identified as an independent poor prognostic factor of both RFS and OS in "intermediate-grade" lung adenocarcinomas. Indeed, patients with the $\mathrm{MP} / \mathrm{S}^{+} \mathrm{Lep}^{-}$ subtype had the worst RFS and OS compared with the other subtypes. Previous studies have not mentioned the effects of the simultaneous presentation of both the MP/ $S$ component (unfavorable prognostic factor) and the Lep component (favorable prognostic factor) on prognosis. The current study found that there were no differences in the 5 -year RFS and OS rates between patients with the MP/ $\mathrm{S}^{+} \mathrm{Lep}^{+}$and MP/S ${ }^{-} \mathrm{Lep}^{-}$subtypes. This finding suggested that the Lep component has a "protective role" which can compensate the detrimental effects of the MP/S component in early-stage lung adenocarcinoma. Compared to the other subtypes, the tumor size of the MP/S ${ }^{-} \mathrm{Lep}^{+}$subtype was significantly smaller. In agreement with the study by Ito and colleagues (11), pT stage was not a significant prognostic factor for either RFS nor OS in this study cohort. Recent studies showed that tumor size is not a universal standard to predict prognosis, especially in stage I lung cancer $(10,17,20)$.

In this study cohort, patients with the MP/S component presented with more pleural invasion, which was consistent with previous reports (21). However, only patients with the $\mathrm{MP} / \mathrm{S}^{+} \mathrm{Lep}^{-}$subtype had a poor prognosis. There was no significant difference in terms of surgical resection procedure between the 4 subtypes $(\mathrm{P}=0.072)$. Patients with the MP/S component were more likely to undergo lobectomy. For the MP/S ${ }^{+} \mathrm{Lep}^{-}$subtype, the prognosis between patients receiving adjuvant chemotherapy and not receiving adjuvant chemotherapy was comparable, suggesting that other treatment modalities are needed to improve the prognosis of this subgroup. However, further studies are required to validate this due to the relatively small sample size in this subgroup analysis.

There were some limitations to this study. First, this was a retrospective study and incomplete data is inevitable. Second, this was a single-institution study and future prospective multicenter clinical trials are warranted to further validate these findings.

\section{Conclusions}

In conclusion, micropapillary and solid patterns as minor components had a negative impact on prognosis. The 
presence of lepidic and micropapillary/solid pathological patterns allows acinar- and papillary-predominant lung adenocarcinomas to be further classified into subgroups with prognostic significance. This classification may be used to evaluate the recurrence risk and guide the adjuvant therapeutic management of these patients.

\section{Acknowledgments}

The authors appreciate the academic support from the AME Lung Cancer Collaborative Group.

Funding: This research was supported by the National Nature Science Foundation of China (Grant No. 81772482); the National Nature Science Foundation of China (Grant No. 81972176); and the Scientific and Innovative Action Plan of Shanghai (Grant No. 20511105204).

\section{Footnote}

Reporting Checklist: The authors have completed the STROBE reporting checklist. Available at https://tlcr. amegroups.com/article/view/10.21037/tlcr-21-934/rc

Data Sharing Statement: Available at https://tlcr.amegroups. com/article/view/10.21037/tlcr-21-934/dss

Conflicts of Interest: All authors have completed the ICMJE uniform disclosure form (available at https://tlcr.amegroups. com/article/view/10.21037/tlcr-21-934/coif). The authors have no conflicts of interest to declare.

Ethical Statement: The authors are accountable for all aspects of the work in ensuring that questions related to the accuracy or integrity of any part of the work are appropriately investigated and resolved. All procedures performed in this study involving human participants were in accordance with the Declaration of Helsinki (as revised in 2013). The retrospective study was approved by the Institutional Review Board of the Shanghai Chest Hospital (No. KS2011). Individual consent for this retrospective analysis was waived.

Open Access Statement: This is an Open Access article distributed in accordance with the Creative Commons Attribution-NonCommercial-NoDerivs 4.0 International License (CC BY-NC-ND 4.0), which permits the noncommercial replication and distribution of the article with the strict proviso that no changes or edits are made and the original work is properly cited (including links to both the formal publication through the relevant DOI and the license). See: https://creativecommons.org/licenses/by-nc-nd/4.0/.

\section{References}

1. Tsao MS, Marguet S, Le Teuff G, et al. Subtype Classification of Lung Adenocarcinoma Predicts Benefit From Adjuvant Chemotherapy in Patients Undergoing Complete Resection. J Clin Oncol 2015;33:3439-46.

2. Travis WD, Brambilla E, Nicholson AG, et al. The 2015 World Health Organization Classification of Lung Tumors: Impact of Genetic, Clinical and Radiologic Advances Since the 2004 Classification. J Thorac Oncol 2015;10:1243-60.

3. Hung JJ, Jeng WJ, Chou TY, et al. Prognostic value of the new International Association for the Study of Lung Cancer/American Thoracic Society/European Respiratory Society lung adenocarcinoma classification on death and recurrence in completely resected stage I lung adenocarcinoma. Ann Surg 2013;258:1079-86.

4. Warth A, Muley T, Meister M, et al. The novel histologic International Association for the Study of Lung Cancer/ American Thoracic Society/European Respiratory Society classification system of lung adenocarcinoma is a stage-independent predictor of survival. J Clin Oncol 2012;30:1438-46.

5. Cox ML, Yang CJ, Speicher PJ, et al. The Role of Extent of Surgical Resection and Lymph Node Assessment for Clinical Stage I Pulmonary Lepidic Adenocarcinoma: An Analysis of 1991 Patients. J Thorac Oncol 2017;12:689-96.

6. Wang $\mathrm{W}, \mathrm{Hu} Z, \mathrm{Zhao} J$, et al. Both the presence of a micropapillary component and the micropapillary predominant subtype predict poor prognosis after lung adenocarcinoma resection: a meta-analysis. J Cardiothorac Surg 2020;15:154.

7. Morales-Oyarvide V, Mino-Kenudson M. High-grade lung adenocarcinomas with micropapillary and/or solid patterns: a review. Curr Opin Pulm Med 2014;20:317-23.

8. Miyahara N, Nii K, Benazzo A, et al. Solid predominant subtype in lung adenocarcinoma is related to poor prognosis after surgical resection: A systematic review and meta-analysis. Eur J Surg Oncol 2019;45:1156-62.

9. Shigenobu T, Takahashi Y, Masugi Y, et al. Micropapillary Predominance Is a Risk Factor for Brain Metastasis in Resected Lung Adenocarcinoma. Clin Lung Cancer 2021;22:e820-8.

10. Tsubokawa N, Mimae T, Miyata Y, et al. Prognostic 
significance of vascular invasion in intermediate-grade subtype of lung adenocarcinoma. Jpn J Clin Oncol 2016;46:1015-21.

11. Ito M, Miyata Y, Yoshiya T, et al. Second predominant subtype predicts outcomes of intermediate-malignant invasive lung adenocarcinoma. Eur J Cardiothorac Surg 2017;51:218-22.

12. Kim M, Chung YS, Kim KA, et al. Prognostic factors of acinar- or papillary-predominant adenocarcinoma of the lung. Lung Cancer 2019;137:129-35.

13. Yanagawa N, Shiono S, Abiko M, et al. The Clinical Impact of Solid and Micropapillary Patterns in Resected Lung Adenocarcinoma. J Thorac Oncol 2016;11:1976-83.

14. Bertoglio P, Querzoli G, Ventura L, et al. Prognostic impact of lung adenocarcinoma second predominant pattern from a large European database. J Surg Oncol 2021;123:560-9.

15. Sica G, Yoshizawa A, Sima CS, et al. A grading system of lung adenocarcinomas based on histologic pattern is predictive of disease recurrence in stage I tumors. Am J Surg Pathol 2010;34:1155-62.

16. Moreira AL, Ocampo PSS, Xia Y, et al. A Grading System for Invasive Pulmonary Adenocarcinoma: A Proposal From the International Association for the Study of Lung Cancer
Pathology Committee. J Thorac Oncol 2020;15:1599-610.

17. Zhu E, Dai C, Xie H, et al. Lepidic component identifies a subgroup of lung adenocarcinoma with a distinctive prognosis: a multicenter propensity-matched analysis. Ther Adv Med Oncol 2020;12:1758835920982845.

18. Bian T, Jiang D, Feng J, et al. Lepidic component at tumor margin: an independent prognostic factor in invasive lung adenocarcinoma. Hum Pathol 2019;83:106-14.

19. Lee G, Lee HY, Jeong JY, et al. Clinical impact of minimal micropapillary pattern in invasive lung adenocarcinoma: prognostic significance and survival outcomes. Am J Surg Pathol 2015;39:660-6.

20. Travis WD, Asamura H, Bankier AA, et al. The IASLC Lung Cancer Staging Project: Proposals for Coding T Categories for Subsolid Nodules and Assessment of Tumor Size in Part-Solid Tumors in the Forthcoming Eighth Edition of the TNM Classification of Lung Cancer. J Thorac Oncol 2016;11:1204-23.

21. Koga K, Hamasaki M, Kato F, et al. Association of c-Met phosphorylation with micropapillary pattern and small cluster invasion in p T1-size lung adenocarcinoma. Lung Cancer 2013;82:413-9.

(English Language Editor: J. Teoh)
Cite this article as: Hou Y, Song W, Chen M, Zhang J, Luo Q, Um SW, Facchinetti F, Bongiolatti S, Zhou Q. The presence of lepidic and micropapillary/solid pathological patterns as minor components has prognostic value in patients with intermediategrade invasive lung adenocarcinoma. Transl Lung Cancer Res 2022;11(1):64-74. doi: 10.21037/tlcr-21-934 

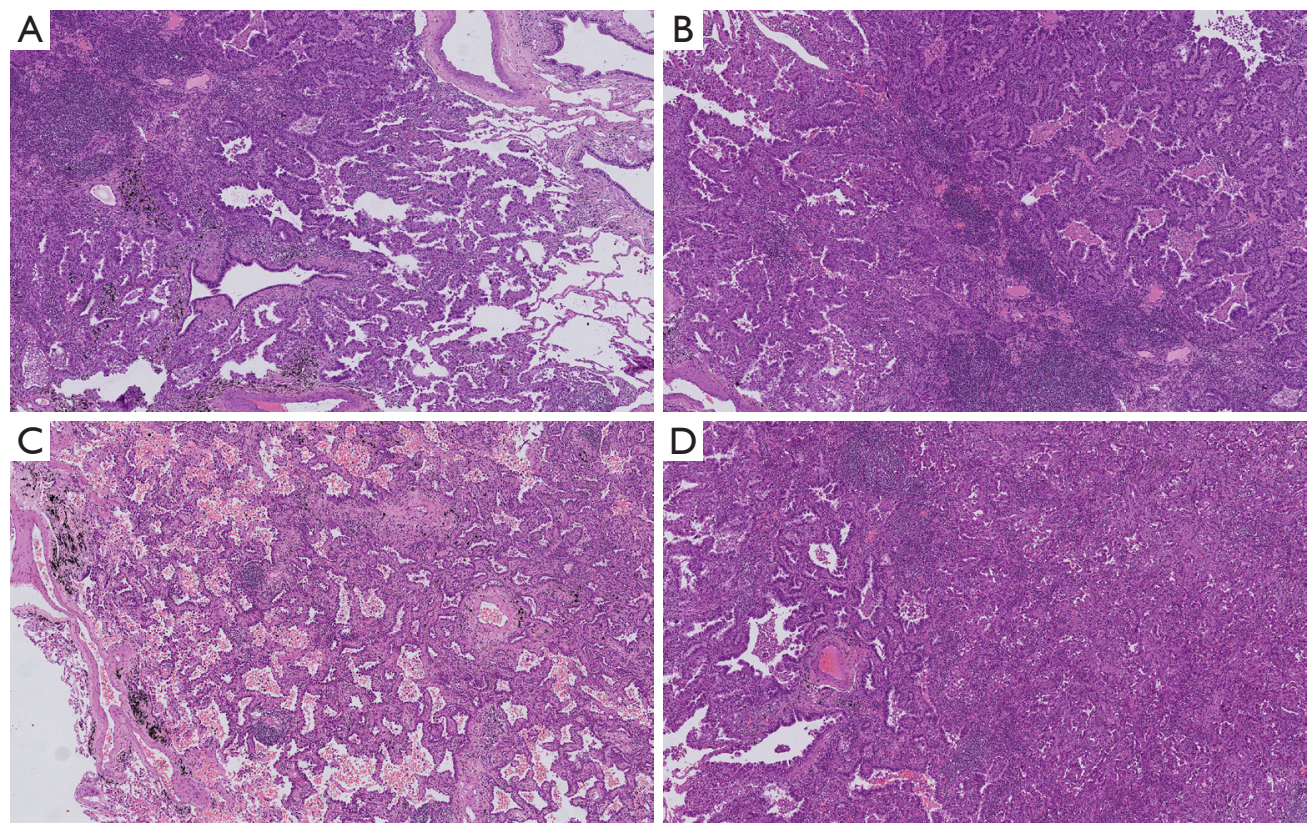

Figure S1 The representative pathological pictures of each subtype. (A) MP/S ${ }^{-} \mathrm{Lep}^{+}$subtype; (B) MP/S $\mathrm{Lep}^{-}$subtype; (C) MP/S $\mathrm{Lep}^{+}$ subtype; (D) MP/S ${ }^{+} \mathrm{Lep}^{-}$subtype. Slides were stained with H\&E stain and images were examined at $\times 50$ objective. 
Table S1 The multivariable analysis results of each subtype as a reference for recurrence-free survival

\begin{tabular}{|c|c|c|c|}
\hline Recurrence-free survival & \multicolumn{3}{|c|}{ Multivariable analysis } \\
\hline \multicolumn{4}{|l|}{ Subtype } \\
\hline MP/S Lep $^{+}$ & Ref & & \\
\hline MP/S'Lep ${ }^{-}$ & 0.954 & $0.366-2.490$ & 0.924 \\
\hline MP/S ${ }^{+} \operatorname{Lp}^{-}$ & 2.457 & $1.096-5.513$ & 0.029 \\
\hline \multicolumn{4}{|l|}{ Subtype } \\
\hline MP/S'Lep ${ }^{-}$ & Ref & & \\
\hline MP/S Lep $^{+}$ & 1.048 & $0.402-2.735$ & 0.924 \\
\hline \multicolumn{4}{|l|}{ Subtype } \\
\hline MP/S'Lep ${ }^{+}$ & Ref & & \\
\hline MP/S'Lep ${ }^{-}$ & 2.658 & $0.849-8.315$ & 0.093 \\
\hline MP/S Lep $^{+}$ & 2.785 & $0.898-8.644$ & 0.076 \\
\hline MP/S ${ }^{+} \operatorname{Lp}^{-}$ & 6.844 & 2.338-20.035 & $<0.001$ \\
\hline \multicolumn{4}{|l|}{ Subtype } \\
\hline MP/S Lep ${ }^{-}$ & Ref & & \\
\hline MP/S'Lep ${ }^{-}$ & 0.388 & $0.166-0.907$ & 0.029 \\
\hline
\end{tabular}

HR, hazard ratio; Cl, confidence; Lep, lepidic; MP/S, micropapillary/solid. 
Table S2 The multivariable analysis results of each subtype as a reference for overall survival

\begin{tabular}{|c|c|c|c|}
\hline Overall survival & \multicolumn{3}{|c|}{ Multivariable analysis } \\
\hline \multicolumn{4}{|l|}{ Subtype } \\
\hline MP/S Lep $^{+}$ & Ref & & \\
\hline MP/Sㄴep ${ }^{-}$ & 2.335 & $0.723-7.540$ & 0.156 \\
\hline MP/S Lep $^{-}$ & 4.045 & $1.285-12.736$ & 0.017 \\
\hline \multicolumn{4}{|l|}{ Subtype } \\
\hline MP/S'Lep ${ }^{-}$ & Ref & & \\
\hline MP/S Lep $^{+}$ & 0.428 & $0.133-1.383$ & 0.156 \\
\hline \multicolumn{4}{|l|}{ Subtype } \\
\hline MP/Sㄴep ${ }^{+}$ & Ref & & \\
\hline MP/Sㄴep ${ }^{-}$ & 4.023 & $1.356-11.937$ & 0.012 \\
\hline MP/S ${ }^{+}$ep $^{+}$ & 1.723 & $0.450-6.593$ & 0.427 \\
\hline MP/S Lep $^{-}$ & 6.968 & $2.261-21.477$ & $<0.001$ \\
\hline \multicolumn{4}{|l|}{ Subtype } \\
\hline MP/S Lep ${ }^{-}$ & Ref & & \\
\hline MP/S'Lep ${ }^{-}$ & 0.577 & $0.240-1.391$ & 0.221 \\
\hline
\end{tabular}

HR, hazard ratio; Cl, confidence; Lep, lepidic; MP/S, micropapillary/solid. 